\title{
Multi-phase Magnetostrictive Actuator Dedicated to Close-Loop Vibration Control System
}

\author{
Jerzy Kaleta ${ }^{1, a}$, Karol Wachtarczyk ${ }^{1, b}$ and Przemysław Wiewiórski ${ }^{1, c *}$ \\ ${ }^{1}$ Wroclaw University of Technology, Department of Mechanics, Materials Science and \\ Engineering, Smoluchowskiego str 25, 50-370 Wroclaw, Poland \\ a jerzy.kaleta@pwr.edu.pl, b karol.wachtarczyk@pwr.edu.pl, \\ c przemyslaw.wiewiorski@pwr.edu.pl
}

Keywords: Magnetostrictive Actuator, Multi-Phase Vibrations, Moving Objects Using Vibrations, Digital Signal Processing, Code Of Structure Stiffness (CSS)

\begin{abstract}
The article discusses a multi-phase magnetostrictive actuator which is the executive part of a multi-phase mechanical vibration controller (MVC). The whole system contains several stages of magnetostrictive actuators and a dedicated vibration sensor. Each individual actuator is dedicated to operation with the same frequency and with an individually programmed phase shift. The controller enables the actuator to excite mechanical vibration in various media (including liquids) to ultrasound frequencies. This allowed to continuously maintain a selected construction in mechanical resonance whose frequency was determined in real time. The work is the continuation of research on the development of the Energy Harvesting $(\mathrm{EH})$ methods by using magnetostrictive actuator - mechanical cross phenomena.
\end{abstract}

\section{Introduction}

The main objective of this work was to construct the multi-phase magnetostrictive actuator forming the executive part of a multi-phase mechanical vibration controller. It was assumed that the proposed solution would enable positioning of objects freely placed on the base (beam) subjected to mechanical vibration. Simultaneously it was assumed that in constructions there is such a form of vibration, up to $30 \mathrm{kHz}$, which allows to supply energy in a controlled way and perform remote mechanical work (e.g. displacement in a set direction, mechanism revolution, unscrewing an element, pressing a switch). For this purpose the idea of generating equal frequency phase-shifted vibration. It involves the excitation of actuators distributed one after another in such a way that subsequently sent signals are shifted with regards to one another by a set angle, which is changed in time in accordance with the adopted algorithm.

Due to the feedback from an integrated vibration sensor, the multi-phase method of shaping mechanical vibration allowed to define and use the so called Code of Structure Stiffness (CSS), which is described below.

However, to achieve the goals the following tasks had to be performed:

- making a prototype of the head of a multi-phase actuator with an integrated vibration level sensor,

- making a dedicated electronic system, vibration controller software and the determination of actuator performance,

- defining the so called Code of Structure Stiffness - CSS,

- developing an original method of objects and mechanisms positioning on the basis of the free displacement of an element along a straight line beam undergoing vibration.

In the summary further development directions were suggested. 


\section{Magnetostrictive exciters of mechanical vibration}

For the purpose of generation of mechanical vibrations is needed to understand of various types of vibrators so called vibration exciters. Their selection depends on vibration level (PSD - Power Spectral Density), frequency and amplitude-phase level of distortion. If it is required that actuator doesn't contain moving parts, the selection is limited to only two types of transducers: Piezoelectric Lead-Zirconate-Titanate (PZT) and a magnetostrictive actuator (most often using Terfenol-D). It should be noted, however, that piezoelectric generators operate only in a precisely determined, narrow resonant frequency range related to the construction of the piezoelectric transducer. A different frequency range results in actuator overheating or PZT rings destruction. On the other hand magnetostrictive actuators operate in a wider frequency range, including also the resonance of the vibrator's construction itself [1] and are characterised by a large generated force (reaching hundreds of $\mathrm{kN}$ ) and high maximum work frequency (up to even $100 \mathrm{kHz}$ ) [2]. Their disadvantage is non-linear characteristic, low vibration amplitude (compare to electrodynamic tranducers) and maximum work temperature, restricted by the acceptable parameters of the induction coil. The price of magnetostrictive actuators is much higher than in the case of typical piezoelectric transducers, hence their use is limited to a small number of applications. However, the predominance of advantages was the reason why magnetostrictive actuators were used in this solution.

The main element in magnetostrictive exciters is the so called magnetostrictive actuator core which is composed of one or more rods, depending on its length, made of materials characterised by giant magnetostrictiction (GMM - Giant Magnetostrictive Materials, e.g. Terfenol-D, cobalt nano-ferrite) and a system adjusting the magnetization level on the basis of neodymium magnets. The number of core components (GMM rods, neodymium magnets, pressure mandrels) is of key significance for its construction, the fewer of them, the higher work frequencies are achieved. One of the solutions used in the core is a system made of alternately located neodymium magnets and Terfenol-D rods [3].

The basic characteristics of materials with giant magnetostriction significantly depend on the parameters of external fields. Due to this the actuator constructions are equipped with permanent magnets generating a bias magnetic field and a spring system causing prestress. The appropriate prestress level allows to increase magnetostrictive amplitude [4,5]. The bias magnetic field in turn increases the linearity of characteristics and sensitivity [6].

The authors have considerable experience in constructing actuators and magnetostrictive actuator harvesters [3], and their earlier research on the use of Terfenol-D have found a number of applications in exciters and vibration transducers $[2,7,8]$.

\section{Construction of vibration level based on a multi-phase magnetostrictive actuator and the Heron system}

The controlled generation of mechanical vibration in a wide range of frequencies was conducted using a multi-phase magnetostrictive actuator with an integrated sensory part. The whole system was controlled with Heron Advanced Multiphase Software which provides control signals to the actuator (frequency and vibration phases) required to maintain the construction in resonance. The vibration controller uses an electronic system containing DSP (Digital Signal Processor) and measurement modules, i.e. input-output modules, DDS (Direct Digital Synthesis) generators and an ICP (Integrated Electronics Piezo-Electric) sensor from PCB Piezotronics. Signal conditioning and increasing level of vibration power is ensured thanks to a dedicated CDM-1P device.

The generation of the so called multi-phase vibration requires a head containing numerous active magnetostrictive parts. In the case of the presented research a head made of four actuators was constructed due to the number of Terfenol-D rods (8 pieces) and the possibility to use 
analogue control as in a typical stepper motor. Moreover, a PCB vibration sensor was used centrally in the system to measure the level of vibration in the excited object. The proposed distribution of actuators and the sensory part is presented in Fig. 1.

a)

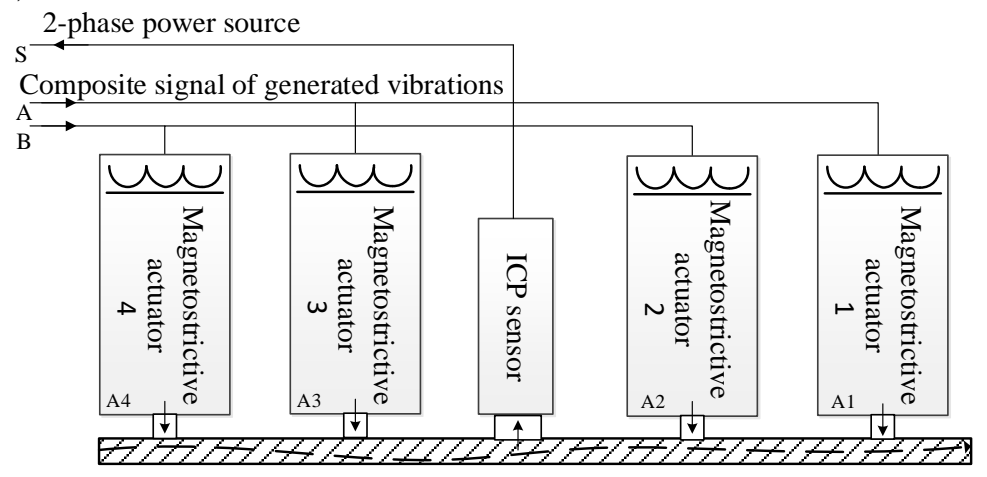

b)

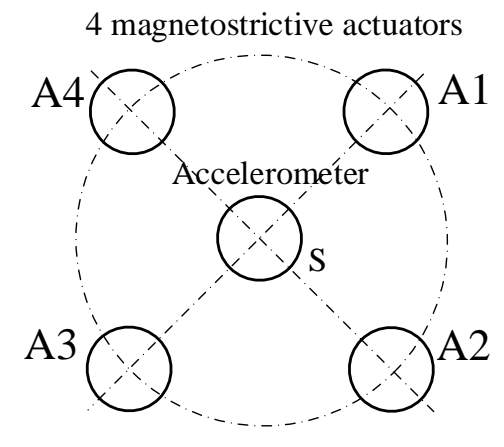

c)

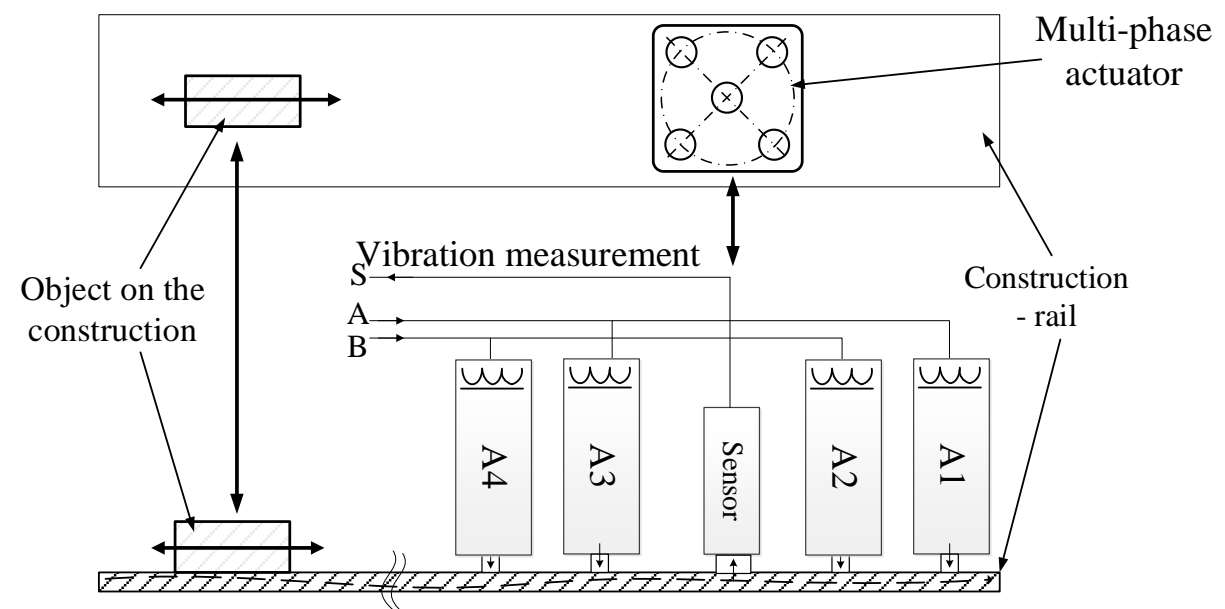

Fig. 1. Idea of the system of four magnetostrictive actuators: a) scheme of actuators operating simultaneously with vibration measurement path; b) symmetrically distributed actuators and central vibration sensor; c) scheme of linear positioning system using vibration

The design and prototype of the head was made in accordance with Fig. 1. Actuators were situated symmetrically (Fig. 1B). Figure 1C shows a scheme of the positioning system using generated vibration with feedback in the case of a straight line bus which is described below.

Figure 2 presents the whole vibration controller system with the designed multi-phase head. Every actuator can be operated separately. It allows to generate even complex mechanical vibrations modes. Apart from the head, the vibration controller system was composed of advanced electronic subsystems based on a Heron card with a floating-point DSP, Texas Instruments C6000 type with extending modules. The system contained also dedicated software using API, Hunt Engineering. 


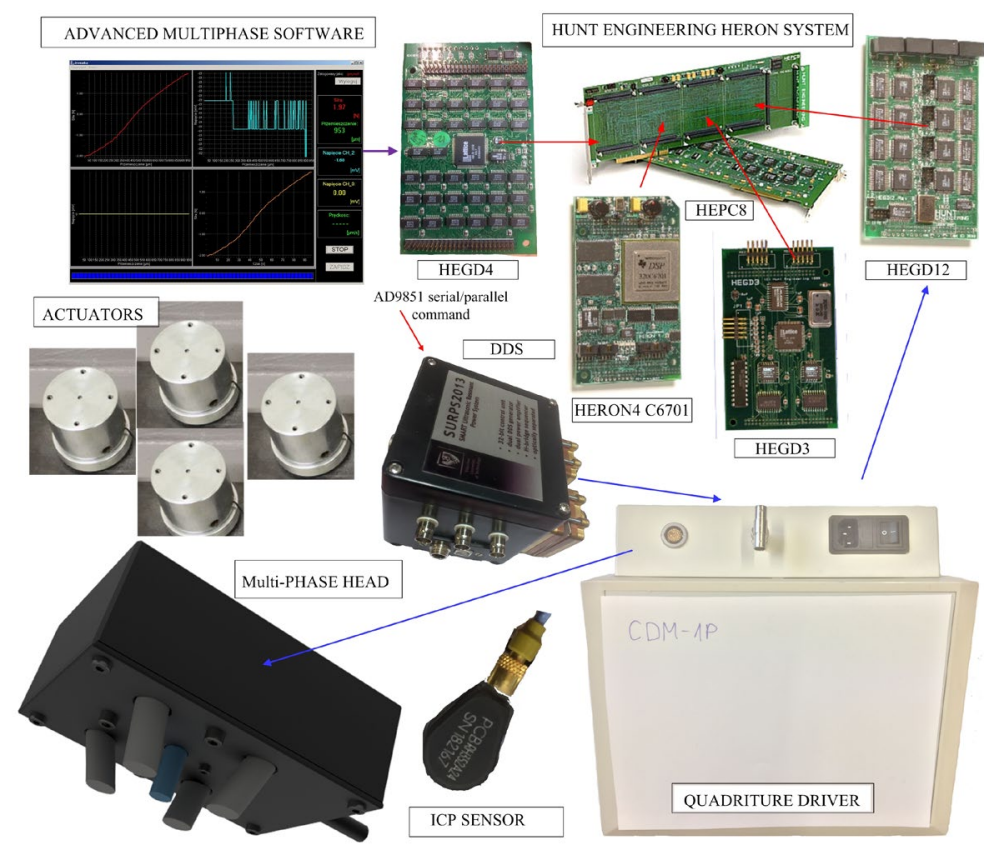

Fig. 2. View of complete vibration controller system based on Hunt Engineering DSP Heron system [9]

System Hunt Engineering HERON was responsible for the signal processing - from acquisition and conditioning the sensor signal to the generation of phase shifted control signals using both DAC (Digital-to-Analog Converter) and DDS stages.

Vibration as electric signals were received by a PCB sensor after appropriate suppling they were collected by a module of 16-bit ADCs - HEGD12. They were used as the basis for the determination of the setpoints of the digital vibration controller which released subsequent DDS values in the feedback loop through HEGD4. The role of CDM-1P was only conditioning (amplification and noise reduction) sensor signals and power amplification for four magnetostrictive actuators.

The Heron Advanced Multiphase Software was to maintain the construction in the resonant state despite the changing resonant frequency. For this purpose, it generated control signals for actuators with the required resonant vibration frequency. The determination of resonant vibration frequency was possible on the basis of the analysis of signal amplitude with a set frequency moment of time. If at a given excitation, the vibration amplitude was decreased, it meant that the system was "leaving" the resonant state. Then the algorithm checked whether after the excitation of the system with frequency higher or lower than the current vibration amplitude it grew. If so, this frequency was considered a new resonant frequency. The amplitude control cycle was repeated.

\section{Remote object positioning using vibration}

A test task used for this system was positioning an object located on the construction using vibration. The task was performed by generating vibration in the range of the mechanical resonance of the construction, and next placing a non-magnetic object, about $30 \mathrm{~g}$, on a vibrating 6-meter long steel beam. Under the influence of mechanical vibration the object was set in motion resulting in subsequent hitting the beam structure. By changing the phase shift between generated signals, it was possible to position the object. The system scheme is presented in Fig. 3A.

The acceleration sensor fixed in the multi-phase actuator head recorded acoustic events occurring at the moment when the object separated from the beam. Its small mass only 
insignificantly changes structure frequency and the evoked acoustic event had significantly higher frequency than the resonant frequency of the beam. Vibration changes were also recorded as a sinusoidal signal with a frequency of $667 \mathrm{~Hz}$. When an aluminium object was placed on the beam, additional acoustic events resulting from the loss of contact between the object and the beam occurred.

\section{Code of Structure Stiffness - CSS}

The acoustic events resulting from hitting the beam with a small mass constitute a characteristic signal reply to of a given structure. They have different amount of energy accumulated in time, characteristic for the beam structure and the adhering element, i.e. small aluminium mass. This response may be a control signal for vibration controller. On the other hand, the possibility to control the vibration level allows to detect changes in the construction.

In other words the number and character of recorded acoustic events is related to the energy supplied to the tested structure in the form of mechanical vibration and the instantaneous state of this structure. Hence a sequence of acoustic events, as a modulated binary run (in accordance with F2F - frequency/double frequency or MFM - modified frequency modulation) will depend on the changing parameters of the medium in which vibration is propagated, including propagation obstacles. The following factors are of key significance here: mechanical structure specific stiffness, number of elements in a structure, induction frequency characteristic for any of the construction elements, material defects, e.g. pores or cracks. This dependence between the energy supplied in the form of vibration to the object and the obtained sequence of acoustic events is called the Code of Structure Stiffness (CSS). In other words, CSS is a characteristic change of frequency response of a complex multi-phase system resulting from the supplied energy. Using the CSS idea and the correct interpretation of the binary signal allows to operate the vibration controller in a wide frequency range that it is possible to relocate an object in a programmable way.

An example of how the CSS method is used is presented in Fig. 3. A series of acoustic events in an ICP sensor signal was distinguished as a result of positioning a shifted object. The essence of the CSS method is the determination of a binary series on the basis of the duration of acoustic events and appropriate the corresponding energy released to the system by the moving object. The amount of this energy is determined by the duration time of the high level form the initiation of a given event. In this way it is possible to obtain a unique code related to the condition of a mechanical construction, which will be the subject of further work.

The number of events was characteristic for the current state of the tested object. With the system of a vibration controller in a wide frequency band, it is possible to identify the dynamic characteristics of a mechanical construction by the classification of its "code" as a result of programmable pure-sinusoidal excitation.

The issue requires more extensive research, however, according to the authors a unique technique for positioning and assessment of objects has been developed. The measurement technique resulting from the method potentially may be used in SHM (Structural Health Monitoring) and also as a competitive method in vibroacoustics. 


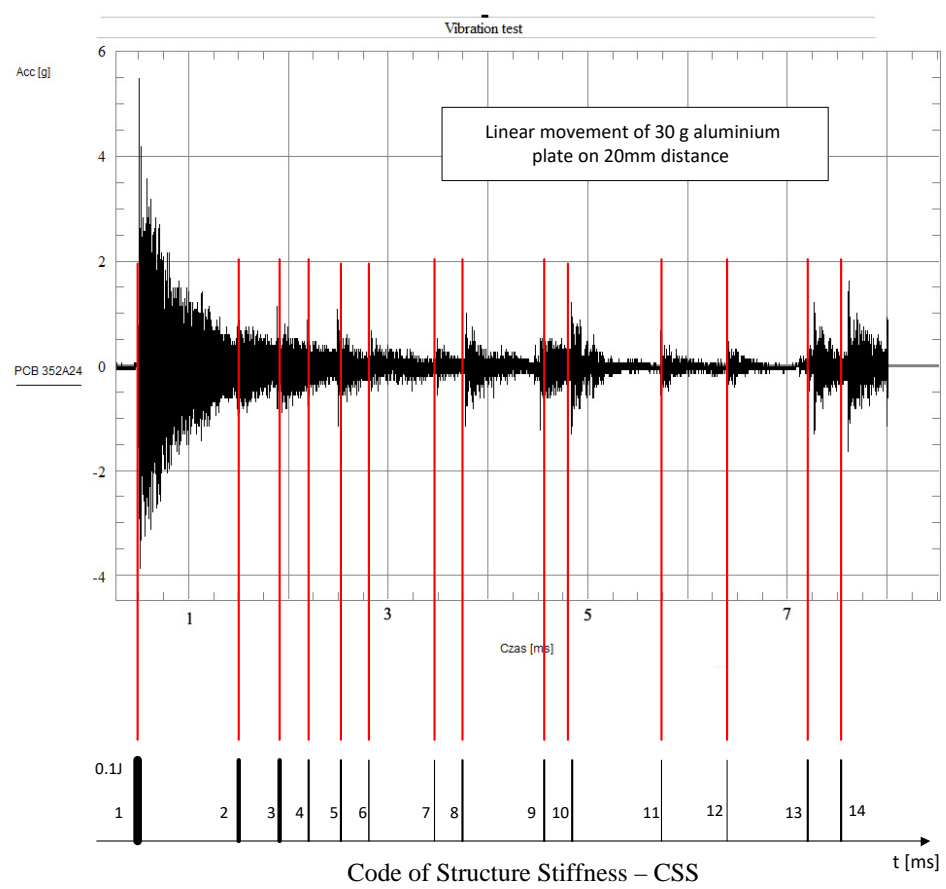

Fig. 3. Decoded signals from ICP sensor as energy stamps of acoustic events in vibration positioning system [9]

\section{Further work directions - changes of the position of medical tools using programmable mechanical vibration.}

According to the authors, the presented method, apart from its use in construction mechanics, can be also used in medicine to set the appropriate position of medical transducers placed in patients' bodies without the necessity for reoperation. Currently there are a number of solutions in which the calibration of medical transducers inside patients' bodies use a few physical phenomena, including: exposure to light of particular wavelength, electric and magnetic field. An example of a mechanism set using an external magnetic field is a valve regulating the level of intracranial pressure in intracranial hypertension (IH) symptoms. After remote programming the valve opening pressure (i.e. the angular position of the mechanism) the correctness of the setpoint is verified with an X-ray image [10].

The proposed development solution in medicine is the use of an acoustic wave with set energy density and a determined frequency band to perform similar "setting" tasks. Thanks to this it will be possible to avoid the influence of a strong magnetic field on mechanism components and also large heat losses which can lead to overheating patient's tissues.

\section{Conclusions}

The main goal of the work has been fully achieved, namely:

- A new generation head prototype was constructed on the basis of four magnetostrictive actuators in a symmetrical system integrated with an accelerometer.

- It was shown that the excitation of resonant vibration using the designed four-step head can be used to set in motion elements located on the surface of the analysed construction by setting the appropriate form of vibration becomes possible by their positioning.

- The so called Code of Structure Stiffness - CSS was defined and used. It was possible thanks to the use of a programmable mechanical vibration controller in a strictly defined frequency range. CSS can be used to classify the behaviour of the components of tested objects. 
In future works the presented results will be investigated with special focus on mechanics application (active vibration damping, energy harvesting with transmission of power and data). One of the main research scopes will be the usage of controlled vibrations made by the designed actuator in NDE diagnostics. The presented method is the expansion of the methods known from Energy Harvesting due to the possibility to transform various forms of energy supplied to/collected from the system for the purpose of performing mechanical work which is object positioning.

The multi-phase actuator system will also be investigated as a potential method to be used in medicine for the positioning of medical devices placed in the patient's body without a need for reoperation. There are already plenty of methods for external calibration of medical transducers in the patient's body, but the proposed method can be used in this situation as well.

Moreover in further steps it will be necessary to pay attention to the costs of material used to make the core of the magnetostrictive actuator. Cores made of Terfenol-D are very expensive and it is legitimate to use another less costly composite replacement which will not decrease actuator parameters.

\section{References}

[1] F. Claeyssen, N. Lhermet, T. Maillard, Magnetostrictive Actuators Compared To Piezoeletric Acutators, 2002.

[2] ETREMA Products, Inc., 2012. Website: http://etrema-usa.com/.

[3] J. Kaleta, R. Mech and P. Wiewiórski, Development of Resonators with Reversible Magnetostrictive Effect for Applications as Actuators and Energy Harvesters, IntechOpen. https://doi.org/10.5772/intechopen.78572

[4] J. Bomba and J. Kaleta, An initial investigation into the change in magnetomechanical properties of Terfenol-D rod due to prestress and temperature, Issue of Material Testers, 2004.

[5] J. Bomba and J. Kaleta, The influence of prestress on magnetomechanical damping in giant magnetostrictive materials, w 20th DANUBIA-ADRIA Symposium on Experimental Methods in Solid Mechanics, Gyor, 2003.

[6] Y. Liang and X. Zheng, Experimental researches on magneto-thermo-mechanical characterization of Terfenol-D, Acta Mechanica Solida Sinica, 2007. https://doi.org/10.1007/s10338-007-0733-X

[7] J. Kaleta, Smart magnetic materials. Structure, manufacturing, testing properties, application (in Polish: Materiały magnetyczne SMART. Budowa, wytwarzanie, badanie właściwości, zastosowanie), Wrocław: Oficyna Wydawnicza Politechniki Wrocławskiej, 2013.

[8] J. Bomba, Damping in giant magnetostrictive material. Experiment, modelling, identification (in Polish: Tłumienie w materiale o gigantycznej magnetostrykcji. Eksperyment, modelowanie, identyfikacja), PhD thesis, supervisor: Jerzy Kaleta, 2010.

[9] J. Kaleta, K. Wachtarczyk and P. Wiewiórski, Multi-phase magnetostrictive actuator dedicated for applications using mechanical vibrations as controlled excitation, Experimental Mechanics of Solids - 28th Symposium, Jachranka, Poland, 2018.

[10] Codman Neuro, Post-operative Programming And X-ray Procedure Guide, 2015. 